\title{
无人机航空摄影测量在 “房地一体”地形测量中的应用
}

\author{
徐志庆 ${ }^{1}$ 刘豪杰 ${ }^{2}$ \\ 1 广东国图勘测地理信息有限公司 2 广东南方数码科技股份有限公司
}

DOI:10.32629/gmsm.v3i4.832

\begin{abstract}
[摘要] 近几年, 无人机技术水平不断提高, 在航天摄影测绘中也日益完善。无人机航天摄影测绘是一种 新型的测绘方法,促使相关从业人员对地理数据的采集朝着全天候、实时化趋势迈进,使之具备长期的使 用效应及经济利益。本文就广东省 “房地一体” 地形测量方面无人机航天摄影测绘的使用进行了详细 探究, 以期可以使之在相关领域得到持续发展。
\end{abstract}

[关键词] 无人机; 航天摄影测绘; “房地一体” 地形地籍测绘; 运用分析 中图分类号: P111.31 文献标识码: A

\section{1 无人机航摄测绘介绍}

无人机航摄测绘属于传统航摄测绘 方式最有效的补充, 其能够补偿传统航 摄测绘方法的不足, 具备灵活简便、迅速 高效、精细精准、应用成本少、适用范 围大等特征。无人机航摄测绘被推广用 在国家大型项目建造、资源挖掘等方面, 特别是在 “房地一地” 地形测量、突发 灾害信息测绘绘制等领域有较大的发展 前景。

\section{1 无人机航摄测绘系统的特征}

无人机航摄测绘系统可以在 “房地 一体”地形测量中获得普遍使用, 主要是 因为无人机航摄测绘系统具有如下特 征: (1)时效性强、性价比高。经过对无 人机航摄测绘与传统卫星绘测相对比, 得知传统卫星绘测信息存档周期很长, 无人机航摄测绘系统可以有效处理这个 问题, 其能够随拍随传, 在短期内完成地 形测绘, 及时为客户带来有效数据资料。 而且, 无人机航摄测绘的价格合适, 相 对于人工测绘, 存在较高的性价比。(2) 基本不受限制。无人机航摄测绘通常是 在低空条件下操作, 对升降场地无过高 要求, 气候环境对它的干扰也很小, 中 国国土面积大、地广物博, 许多地方长 期受恶劣自然环境因素的影响, 因为无 人机不受高度的过多制约, 成图的清晰 率也很高, 能够在很大程度上处理这些 问题。(3)快速获得数据资料完成模型创
建。无人机航摄测绘选择了许多高技术 数码摄影系统, 可以及时、精准、迅速的 形成DOM、DSM等信息影像和3D可视化信 息、模型等。

\section{2 无人机航摄系统对 “房地一} 体”地形测量

无人机航摄测绘系统是一种非常关 键的测量方法, 其能够把GPS定位软件内 的导航数据和无人机航摄影像信息相结 合, 进而获得对测量范围中影像及地形 地貌信息。利用无人机航摄测绘系统, 可以直接记录和存储地形测量结果。无 人机在航空测绘地形条件时, 通常是像 控点确定在比较明显的地方来获取更好 的信息。空中三角测绘是借助无人机航 摄系统来测量地形条件的一个非常关键 的内容, 其基本原理在于借助无人机测 绘所测地方的地形地貌, 采集其中有用 数据。在整个地形测量环节, 系统可以按 照所测地方的实际状况, 合理调节测绘 仪器, 但在开展地形的三角测绘以前, 也 要求人工调整测绘参数, 确保不同衔接 点更满足地形测量项目需求。

\section{3 “房地一体” 地形地籍测量} 方面无人机航天摄影测绘技术的运 用分析

3. 1 基于专业理论确定像控点

像控点属于测图与摄影测绘管理加 密的前提条件, 所以指示点位的精准性 与像控点目标选取的好坏将直接影响到
测量结果的真实度。因此, 基于专业理论 科学设立像控点是十分重要的。能在两 条及之上的航线周围朝重合中线周边设 立平高控制点, 而且采取明显物体点刺 点与安装控制标志的手段在开展摄影拍 摄以前完成实地选点。刺点规范要参照 如下条件展开挑选, 比如水渠、街道交叉 位置, 房屋、围墙拐角位置等, 另外就是 以高程改变很小的位置视为高程像控点 的刺点选取标准。

项目过程中, 利用测区 $1: 2000$ 数字 正射影像图, 在室内预先选择成图条件 较好的地物点, 通过实地踏勘确定点位, 每 $100 \mathrm{~m}-150 \mathrm{~m}$ 布设一个像控点, 以提高解 析精度。所有的像控点都需要制作像控 点点位信息表, 同时拍摄点位照片, 以便 后期检查、复测, 每个点分别需要拍摄远 景、中景、近景, 远景和中景宜分不同方 向拍摄, 反映像控点周围情况。

\section{2 采取空中三角测绘方法测绘}

依靠摄影目标当中的空间几何联系 和航天摄影影像, 利用少数像控点来计 量测绘待求点的高度、影像外方位要素、 平面部位等内容的方法就是空中三角测 绘, 项目过程中采取解析法研究空中三 角测绘, 作业流程图如下图所示。此外单 独模型法、光束法以及航带法等均是空 中三角测绘典型的建网手段。航摄仪产 品和POS相融合在无人机空中三角测绘 方面得以实现, 而且借助GPS系统定位航 
摄器, 进而获取其无人机姿态参量与具 体部位的有关参数。采取科学方法处置 IMU与DGPS信息以后就获得了相关航摄 中的 6 个外方位要素。但是, 在进行航摄 测绘活动时, 利用计算机系统和智能化 测定系统与无人机完成连接的形式, 就 可以对有关航摄的信息展开精准的计算 处理与分析。在整个航摄过程无需任何 人为干预, 但是为了便于以后测绘工作 的顺利进行, 在采取空中单脚测绘方法 确定测绘目标的部位时, 要求相关工作 人员选择恰当的测定衔接点, 采取科学 调试方法来处理确定的像控点与衔接点, 可以有效完成空中三角测绘。

3.3 无人机航摄测绘成果验收和信 息采集

在测量时经联合使用空中三角测绘 加密成果与数字摄影测绘进而获得精准 的空中三角测绘成果, 同时采取科学方法 复原立体模型且创建与之有关的数字系 统, 并利用相关软件采集房地项目相关要 素数字线划图及生成DOM正射影像图。

经过对相关摄像摄影的判断和合理 的区域影像特征图加以分析, 来确定影 像所代表地表地物的属性状况和相关特 征。在判定影像时能够采取整体判读调 绘法处理, 整体判读调绘法主要指先室 内完成判读调绘, 然后户外完成定性、检 核、调研, 最后清绘与整饰。

另外, 在测量成果验收中借助航摄 影像所绘地形地物点信息资料和户外 实测信息成果展开质量检测, 并按照有 关要求, 利用通过的数字线划图、正射 影像图等资料进行 “房地一体” 地籍调 查工作底图的制作。在检测居民地的房 屋结构和楼层信息, 可以通过立体模型 获得, 也可以通过外业调绘进行检测, 同时对相关标记是否规范、居民地轮廓 特征是否显著、有关支线与主次干道是 否明确、有关要素性质是否完善等展开 仔细检查。最后基于相应的成图系统来 编辑处理所获取的测量数据后获得整 饰图。

而对于DLG数字线划图质量精度的 验收, 则使用RTK采取CORS系统的户外测 绘方法确定每个明显地物点, 项目中在

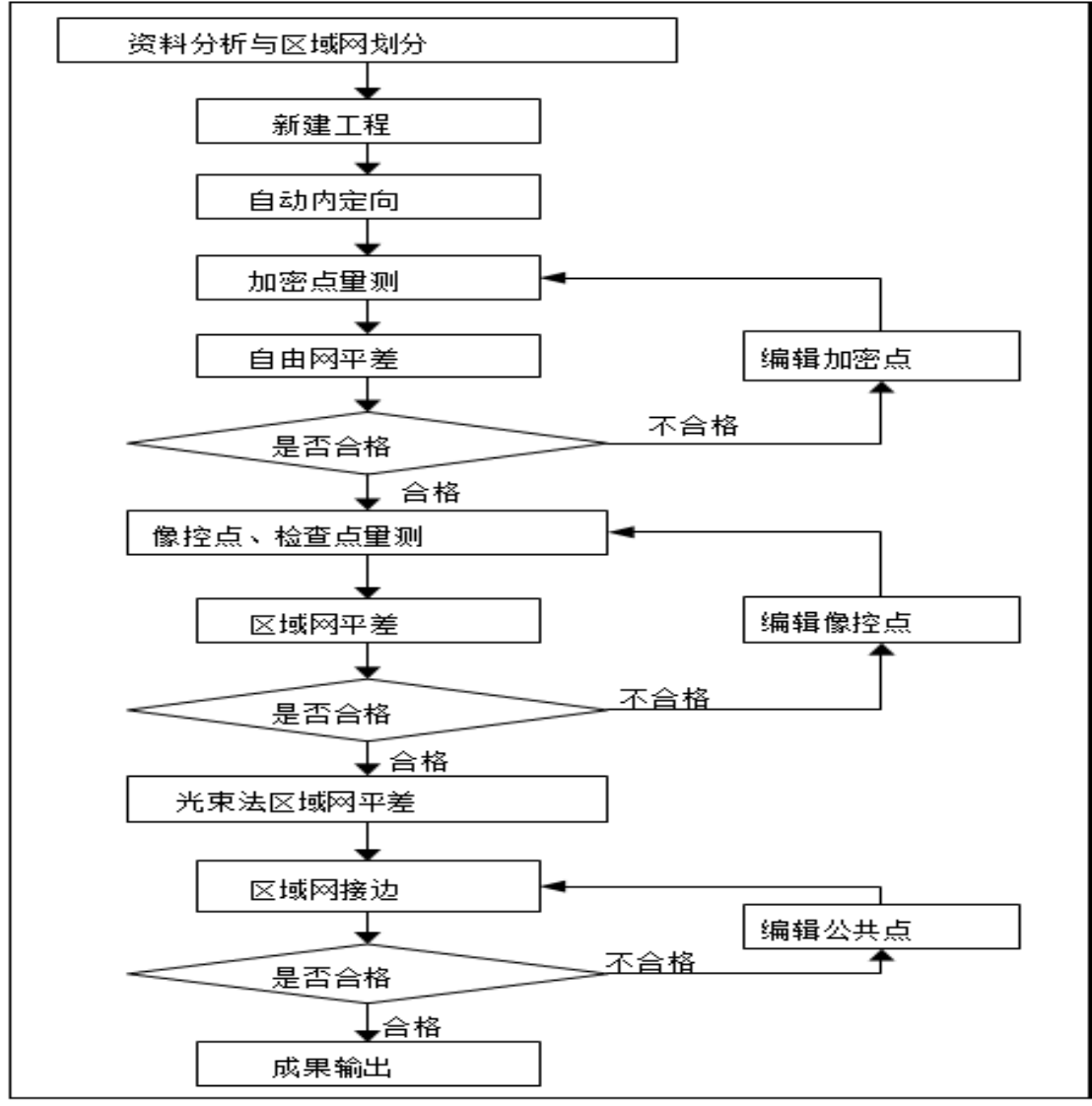

空中三角测量作业流程图

精度统计表

\begin{tabular}{|c|c|c|c|}
\hline 点位精度区间分布 & 个数 & 百分比 & 备注 \\
\hline $0 \leqslant \triangle<0.05 \mathrm{~m}$ & 215 & $80.83 \%$ & \multirow{3}{*}{$\begin{array}{c}\text { 碎部点检查 } 266 \text { 点, 最大 } \triangle X=0.143 \mathrm{~m} \text {, 最大 } \triangle \\
Y=0.135 \mathrm{~m} \text {, 最大 } \triangle S=0.124 \mathrm{~m} \text {, 粗差率为 } 3.01 \% \text {, 小 } \\
\text { 于 } 5 \% \text {, 符合要求; 剔除超限点位计算中误差, 得点 } \\
\text { 位中误差为 } \pm 0.042 \mathrm{~m} \text {, 小于 } \pm 0.05 \mathrm{~m} \text {, 满足要求 }\end{array}$} \\
\hline $0.05 \mathrm{~m} \leqslant \triangle<0.10 \mathrm{~m}$ & 43 & $16.17 \%$ & \\
\hline $0.10 \mathrm{~m} \leqslant \triangle$ & 8 & $3.01 \%$ & \\
\hline
\end{tabular}

图上选取二百六十六个明显的地物点进 行, 包含了相应的街道交叉处、围墙和房 角等。根据《“房地一体” 农村宅基地和 集体建设用地权籍调查技术指南 (试 行) 》中的精度要求, 计算结果为粗差率 $3.01 \%$ (小于 $5 \%$ ); 剔除超限点位计算中误 差, 得点位中误差为 $\pm 0.042 \mathrm{~m}$ (小于 \pm $0.05 \mathrm{~m})$, 满足要求, 因此航摄测绘获得 DLG成果通过整体判定, 结果是 “合格”, 精度统计表如上:

\section{4 结束语}

总之, 在测量多种地形时, 选择无人 机航摄测绘可以有效提升工作效率。而 且, 经过合理使用无人机航摄测绘系统, 可以获取精准、高效的清楚倾斜影像与 正射影像, 且基于此, 利用相关的成图系 统来处理数字资料, 由此完成对 $3 \mathrm{D}$ 数字
模型与正射影像图的制作。所以, 无人机 航摄测绘方法在提升 “房地一体” 地形 地籍测绘精度与绘制效率上均有不可忽 略的功能。

\section{[参考文献]}

[1]贺波,李海燕.无人机倾斜摄影测 量在建筑规划竣工测绘中的应用 $[\mathrm{J}]$. 科 学技术创新,2020,(25):66-67.

[2]杨亚樹,谢思梅,谢荣安.无人机 倾斜摄影测量技术在不动产更新测绘中 的应用[J].测绘通报,2020,(7):108-111。

[3]傅成栋.无人机航空数字测量技 术在地形测绘中的应用 [J]. 数字技术与 应用,2020,38(07):95-96.

[4]姜华.无人机测量技术在地形 测量方面运用分析 [J]. 数字技术与应 用,2020,38(05):111-112. 